\title{
Rotating black holes with magnetic fields as accelerators of charged particles
}

\author{
C. H. Coimbra-Araújo* and R. C. Anjos ${ }^{\dagger}$ \\ Departamento de Engenharias e Exatas, Universidade Federal do Paraná, Pioneiro, 2153, \\ 85950-000, Palotina, Brazil \\ E-mail: carlos.coimbra@ufpr.br
}

\begin{abstract}
In the present work we review the circumstances under which charged particles can escape black hole gravitational fields after its collision with another particle. Here we examine the dynamics of neutral and charged particles interacting with slowly and rapidly rotating Kerr black holes in the presence of magnetic fields. In this respect, rotating black holes may act as particle accelerators under certain circumstances. Axially symmetric magnetic field homogeneous at infinity and other type of configurations are discussed. It is analyzed the effective potential of the system, as well the center of mass energy and the escape velocity of the particles. As appointed by many examples in the literature, some of the main results point that the intensity of the magnetic fields induces the innermost stable circular orbit (ISCO) to be closer to the black hole horizon. There is a range of possible values for the spin $a$ and for the magnetic field such that particles should escape from the system. The present investigation on particle escape velocity can possibly contribute, for example, to constrain AGN dynamic properties to properly understand the acceleration mechanisms of the highest energy cosmic rays in the universe.
\end{abstract}

International Conference on Black Holes as Cosmic Batteries: UHECRs and Multimessenger Astronomy BHCB2018

12-15 September, 2018

Foz do Iguaçu, Brasil

\footnotetext{
* Speaker.

${ }^{\dagger}$ This work has been supported by .
} 


\section{Introduction}

Black holes (BHs) are extremal objects with very specific physical characterists. They were predicted by general relativity (GR) and have been observed by quite a few astronomical measurements as optical and X-ray observations and also gravitational wave detection. In this respect, there is a current endeavor among physicists and astronomers to answer the question if GR and astrophysical BHs are indeed the same thing.

GR black holes are defined by literature "as a region of the spacetime in which gravity is so strong that nothing, nor even light, can escape. The event horizon is the boundary of such a region" [5]. This region of spacetime "cannot communicate with the external universe" [25] and indeed "nothing can be blacker than a black hole" [22].

On the other hand, stellar or supermassive astrophysical black holes are connected to the inusitated dynamics of stars in the center of galaxies in general; the activity observed in the center of Active Galactic Nuclei (AGNs); the phenomena called 'microquasars' and 'collapsars'. Fundamentally, astrophysical BHs have some of the Kerr BH properties, i.e., at least it appears that astrophysical BHs have mass and spin [24, 14]. In this respect, observations show that several stellar or supermassive BHs have high spin $(a>0.7)[6]$.

High energy astrophysical phenomena indicate that possibly BHs can be viewed as cosmic batteries. For example, it is commonly accepted that relativistic jets from central BHs of AGNs can grant the common origin of the ultrahigh energy cosmic rays (UHECRs) in the universe. This role played by such astrophysical BHs is becoming a prominent scientific issue in the so called multimessenger astronomy. Earth laboratories attached to several scientific collaborations are more and more concerned to investigate the interplay between cosmic ray observation and black hole activity in the center of galaxies. This is important since the provenance of the UHECRs is an open half-century-old conundrum [20].

The Hillas condition conveys that AGNs are capable of accelerating a $Z$ charge particle to a maximum energy $Z \approx 10^{19} \mathrm{eV}$ in jets or at external shocks that are recognized to be sites of leptonic emissions [17, 23].

In general, the gravitational dynamics of charged (or neutral) particles around BHs could shed some light on the roots of the high energy phenomena associated to jets (which involve escaping particles) or accretion disks (particles in circular orbits). The presence of strong gravitational and electromagnetic fields deviates charged particles from stable orbits, inducing the appearance of inter-particle collisions. Additionally, a rotating BH may provide sufficient energy to the particle moving around it and, in some specific cases, the particle may escape to infinity. Many astrophysical evidences point that a magnetic field might be present. Aliev and Ozdemir [2], and other authors (for a review see $[18,16]$ ) studied the dynamics of particles orbiting Kerr BHs with weakly magnetized black holes. Astrophysically, the origin of such magnetic field is explained from the existence of a plasma in the proximity of the $\mathrm{BH}$ (e.g. astrophysical accretion disks) [21].

The present review introduces some aspects concerning the acceleration of neutral or charged classical particles near the horizon of static or rotating black holes, in the presence or not of magnetic fields. In what follows, we use the $(-+++)$ metric signature and natural units (where $G=c=1)$. 


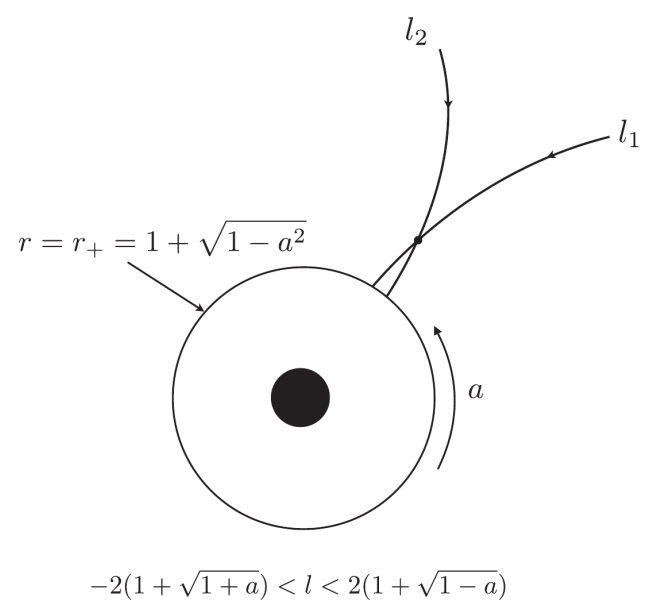

Figure 1: Schematic figure of particle collision (neutral or charged) in the vicinity of a rotating black hole. Figure from [7]. The allowed range of particle angular momenta for geodesics falling into the $\mathrm{BH}$ is also given.

\section{General properties of test particles near a black hole}

Some exterior solutions of the Einstein equations in vacuum could be interpreted as black holes with some particular properties. For example, it can be mentioned three important solutions:

I) The Schwarzschild solution (static solution):

$$
d s^{2}=-\left(1-\frac{2 M}{r}\right) d t^{2}+\left(1-\frac{2 M}{r}\right)^{-1} d r^{2}+r^{2}\left(d \theta^{2}+\sin ^{2} \theta \phi^{2}\right),
$$

where $M$ is the gravitational mass of the static black hole.

II) The Reissner-Nordström solution (static solution with an electric charge q):

$$
d s^{2}=-\left(1-\frac{2 M}{r}+\frac{q^{2}}{r^{2}}\right) d t^{2}+\left(1-\frac{2 M}{r}+\frac{q^{2}}{r^{2}}\right)^{-1} d r^{2}+r^{2}\left(d \theta^{2}+\sin ^{2} \theta \phi^{2}\right),
$$

which recovers the Schwarzschild solution in the particular case of a neutral black hole $(q=0)$.

III) The Kerr solution (vacuum BH solution with rotation):

$$
\begin{aligned}
d s^{2}= & -\left(1-\frac{2 M}{\Sigma}\right) d t^{2}-\frac{4 a M r \sin ^{2} \theta}{\Sigma} d t d \theta+\frac{\Sigma}{\Delta} d r^{2} \\
& +\Sigma d \theta^{2}+\frac{\left(r^{2}+a^{2}\right)^{2}-a^{2} \Delta \sin ^{2} \theta}{\Sigma} \sin ^{2} \theta d \phi^{2}
\end{aligned}
$$

where $M$ is the gravitational mass of the black hole and $a$ is its angular momentum normalized by mass, $\Sigma=r^{2}+a^{2} \cos ^{2} \theta$ and $\Delta=r^{2}+a^{2}-2 M r$. The event horizon is located at $r_{H}=$ $M+\sqrt{M^{2}-a^{2}}$. The ergoregion is described by $r_{H}<r<r_{E}(\theta)=M+\sqrt{M^{2}-a^{2} \cos ^{2} \theta}$. Fig. 2 
illustrates such main regions. The Kerr solution is reduced to the Schwarzschild solution when $a=0$. The last stable circular orbit around Kerr BHs is called ISCO (inner stable circular orbits, see Fig. 3). The inner circular orbit that a test particle could orbit before falling into the horizon is also represented in Fig. 3. The influence of spin in positioning the ISCO is showed in Fig. 4: the greater the spin $a$, the more displaced the minimum of the potential is. This displacement occurs in the direction of the horizon, indicating that the ISCO is closer to the horizon as the value of the spin (a) approaches 1. For a test particle of mass 1, this potential is calculated from radial geodesics as $\frac{1}{2} \dot{r}^{2}+V(r)=0$.

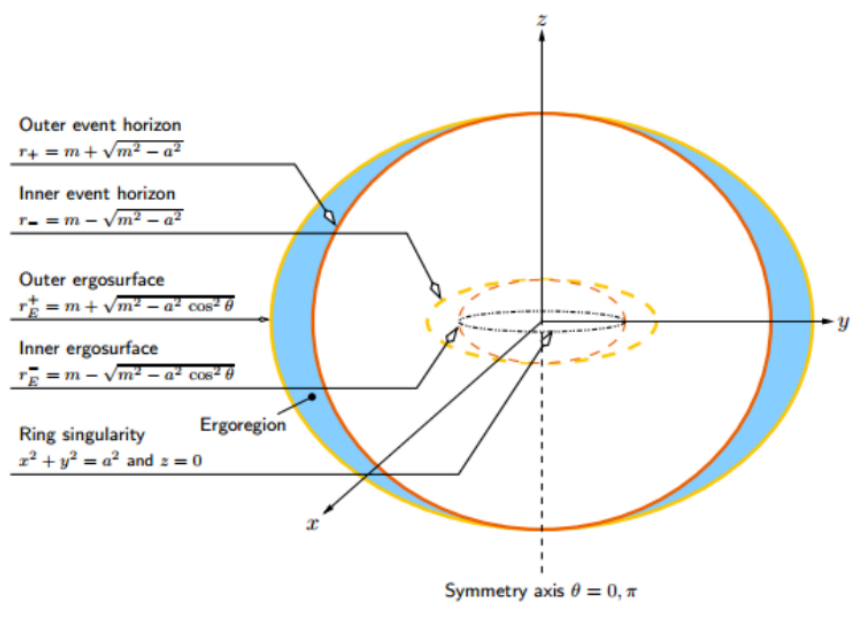

Figure 2: Schematic representation of the Kerr horizons and ergosurfaces.

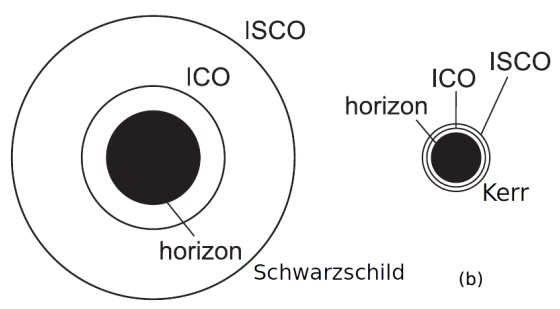

Figure 3: The ISCO and the ICO. Note that the ISCO tends to approach the ICO in the Kerr BH case.

When two neutral or charged particles approach the horizon of a black hole, since the background is curved, it is necessary to define the center-of-mass frame properly. The center-of-mass energy of the two particle system is given by [7]

$$
E_{c . m .}=m_{0} \sqrt{2} \sqrt{1-g_{\mu \nu} u_{(1)}^{\mu} u_{(2)}^{v}},
$$

where $m_{0}$ is the mass of the two colliding particles and $u_{(1)}^{\mu}$ and $u_{(2)}^{v}$ are the 4-velocities of each particle, properly normalized by $g_{\mu \nu} u^{\mu} u^{v}=-1$. The main conditions for accelerating the colliding particles are regarded from the BH spin, i.e. it is awaited that Schwarzschild BHs would not be 


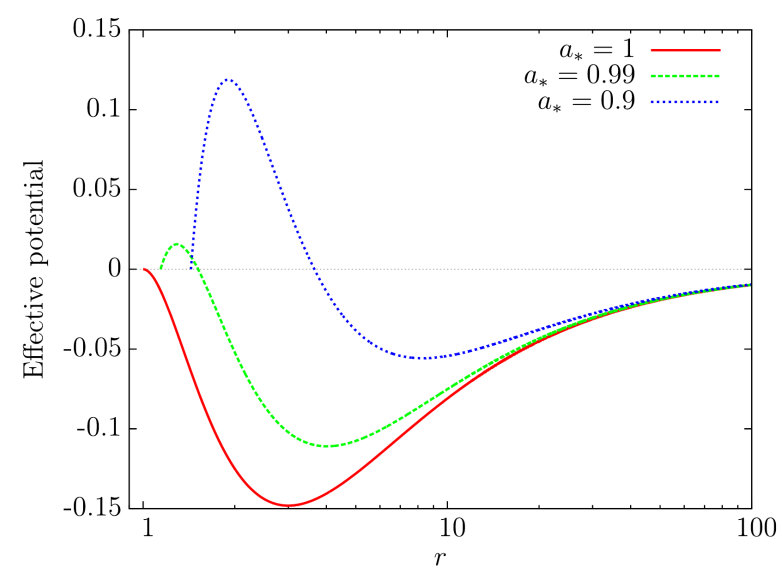

Figure 4: Effective potential for marginally bound critical particles. The potential must be nonpositive in the allowed region of particle motion. The minimum describes where the ISCO is located for some possible spins $a$. From [16].

good accelerators since as told ISCO will not approach ICO and the collision will occur far from the horizon; the angular momentum of particles; the presence of magnetic fields (and in this last case, it is awaited that charged particles would be more accelerated than neutral particles).

\section{Schwarzschild black holes (neutral particles and no magnetic fields)}

The Schwarzschild black hole represents the vacuum solution of Einstein equations outside a spheric symmetric static configuration. For this case the line element is described by equation (2.1). Given the Killing vector $\xi_{(t)}=\xi_{(t)}^{\mu} \partial_{\mu}=\frac{\partial}{\partial t}$ related to the free test particle energy conservation $\left(E=-g_{t \mu} p^{\mu}\right)$ and the Killing vector $\xi_{(\phi)}=\xi_{(\phi)}^{\mu} \partial_{\mu}=\frac{\partial}{\partial \phi}$ related to the free test particle angular momentum conservation $\left(L=-g_{\phi \mu} p^{\mu}\right)$, it is possible to calculate the solution to the geodesic equation (see e.g. [7])

$$
\dot{t}=\left(1-\frac{2 M}{r}\right)^{-1}, \quad \dot{r}=-\frac{1}{r^{2}} \sqrt{r\left(2 r^{2}+2 L^{2}-r L^{2}\right)}, \quad \dot{\phi}=\frac{L}{r^{2}} .
$$

Here $\dot{x}=d x / d \tau$, the derivative with respect to the proper time. Making $L=l_{1}$ and $L=l_{2}$ for the angular momentum of each particle, and $m_{0}=1$, the center-of-mass energy is calculated as

$$
E_{c . m .}=\left[\frac{2 r^{2}(r-1)-l_{1} l_{2}(r-2)-\sqrt{2 r^{2}-l_{1}^{2}(r-2)} \sqrt{2 r^{2}-l_{2}^{2}(r-2)}}{r^{2}(r-2)}\right]^{1 / 2}
$$

Fig. 5 shows the behavior of the radial geodesics and the center-of-mass energy for the Schwarzschild case.

As told before, Fig. 5 shows that Schwarzschild BHs would not be good accelerators. The calculation of stable orbits in this case shows that the inner stable orbit is locate in $r=6 M$, from the horizon. 

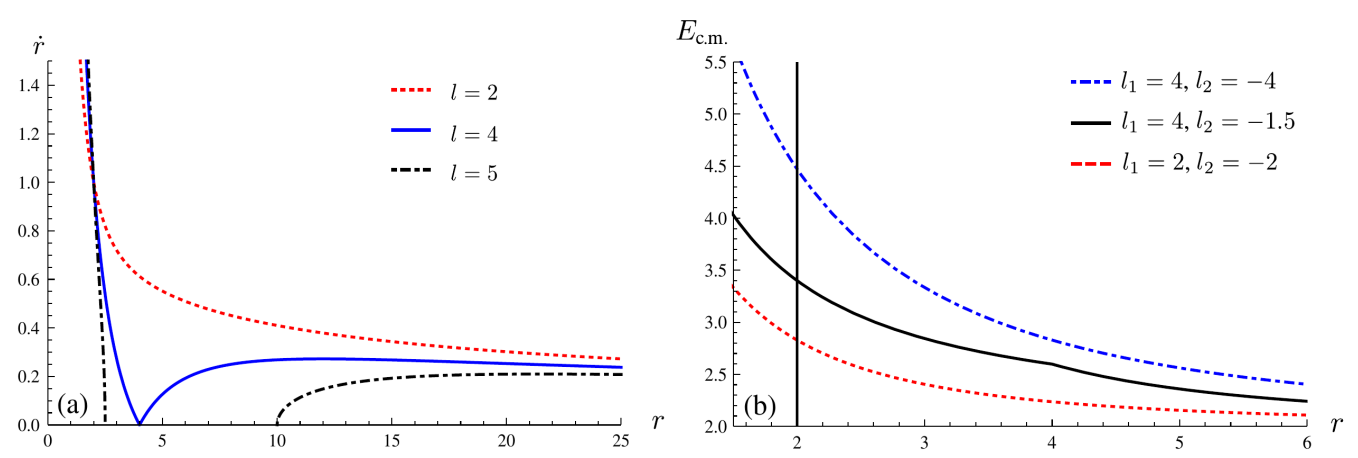

Figure 5: (a) The radial geodesics $\dot{r}$ of neutral particles for a Schwarzschild black hole with no magnetic fields for some values of the particle angular momentum. (b) The variation of the center of mass energy of two particles colliding close to the horizon for some values of the particle angular momentum. Figure from [7].

\section{Kerr black holes}

\subsection{Neutral particles and no magnetic fields}

We consider the motion of neutral particles around rotating black holes described by the Kerr metric in the Boyer-Lindquist coordinates, i.e., the line element (2.3). This represents a stationary vacuum black hole. Given the Killing vector $\xi_{(t)}=\xi_{(t)}^{\mu} \partial_{\mu}=\frac{\partial}{\partial t}$ related to the free test particle energy conservation $\left(E=-g_{t \mu} p^{\mu}\right)$ and the Killing vector $\xi_{(\phi)}=\xi_{(\phi)}^{\mu} \partial_{\mu}=\frac{\partial}{\partial \phi}$ related to the free test particle angular momentum conservation $\left(L=-g_{\phi \mu} p^{\mu}\right)$, it is possible to calculate the solution to the geodesic equation (see e.g. [7]) of neutral particles near black holes with no magnetic fields. At the equator axis of the configuration (i.e. $\theta=\pi / 2$ ) the geodesics of $m_{0}=1$ particles are calculated as

$$
\begin{aligned}
\dot{t} & =-\frac{1}{r^{2}}\left[a(a E-L)+\left(r^{2}+a^{2}\right) T / \Delta\right], \\
\dot{r} & \left.=\frac{1}{r^{2}} \sqrt{T^{2}-\Delta\left[r^{2}+(L-a E)^{2}\right.}\right], \\
\dot{\phi} & =-\frac{1}{r^{2}}[(a E-L)+a T / \Delta],
\end{aligned}
$$

where $T=E\left(r^{2}+a^{2}\right)-L a$ and $\Delta$ is defined in equation (2.3). The center-of-mass energy of the system of two-particles is calculated from (4.1) and from normalization $g_{\mu \nu} u^{\mu} u^{v}=-1$. When $r \rightarrow r_{+}$and $a=1$, this energy is

$$
E_{c . m .}=\sqrt{2\left[\frac{l_{2}-2}{l_{1}-2}+\frac{l_{1}-2}{l_{2}-2}\right]} .
$$

Fig. 6 shows the geodesics for some values of $a$ and the effect of the two-particle angular momenta in the center-of-mass energy. If $l_{1}$ and $l_{2}$ are fine-tuned to some critical value implies in the feasibility of accelerating particles to high energies, making them possible to escape to infinity. 

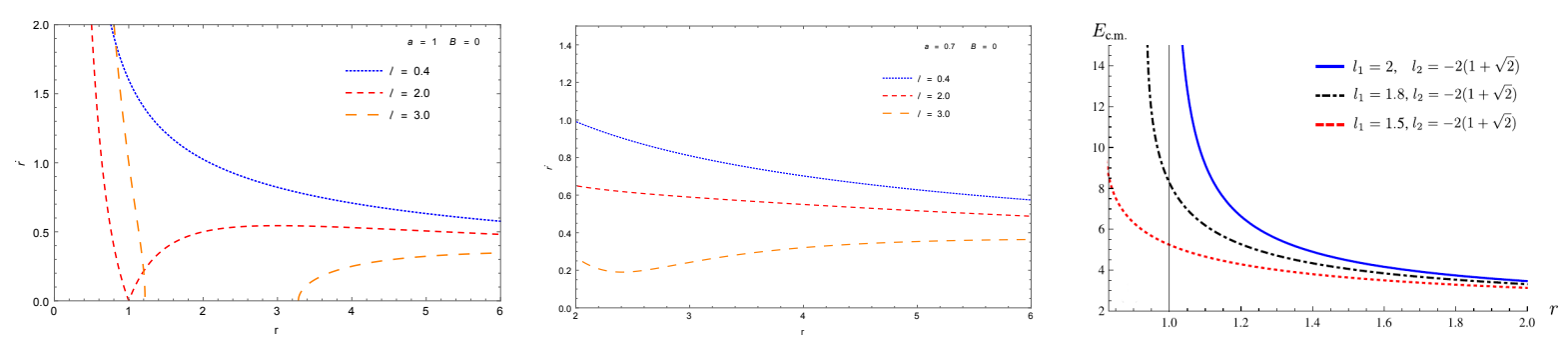

Figure 6: Left: radial geodesics for neutral particles in the vicinity of a extremal Kerr BH $(a \rightarrow 1)$. Middle: the same but for $a=0.7$. For both cases, $B=0$. Right: Center-of-mass energy near the horizon $r_{+}$for some values of $l_{1}$ and $l_{2}$ when $a$ approaches 1. Figure from [13] and [7].

\subsection{Slowly Kerr black holes (charged particles with magnetic fields)}

Hussain et al [18] investigated the dynamics of a charged particle moving around a slowly rotating Kerr black hole in the presence of an external magnetic field. They were seeking the conditions under which charged particles escape from slowly Kerr BHs.

It is assumed that magnetic fields do not disturb the geometry of the BH but they could change the motion of the charged particles that are orbiting the $\mathrm{BH}[28,9]$. It is admitted that there are strong magnetic fields around the central black holes of AGNs (magnetic flux estimated to $\sim 10^{4}$ Gauss). The non-dimensional ratio $b$ of the Lorentz force to the gravitational force is estimated as [16]

$$
b=\frac{q B G M}{m c^{4}} \sim 10^{11}\left(\frac{q}{e}\right)\left(\frac{m}{m_{e}}\right)^{-1}\left(\frac{B}{10^{8} \mathrm{Gauss}}\right)\left(\frac{M}{10 M_{\odot}}\right),
$$

where $B, q, e$ and $m_{e}$ are the magnetic flux density, the charge of the particle, the elementary charge and the electron mass, respectively, and $G$ and $c$ are restored. For an extremal $(a \rightarrow 1)$ Kerr BH immersed in an uniform magnetic field, the center-of-mass energy is estimated as $E_{c . m .}(b) \approx$ $3^{-0.25} \sqrt{b} E_{c . m .}(0)$, where $E_{c . m .}(0)$ is the center-of-mass energy in the absence of magnetic fields.

When a charged particle is orbiting in the innermost stable circular orbit (ISCO) of a Kerr BH it could be abruptly hit by a incoming neutral particle. The energy of this incoming particle leads to three possible results: the charged particle is ejected to infinity, or it could be captured by the $\mathrm{BH}$, or it remains orbiting in the ISCO.

For example, Hussain et al. approach [18] considers a slowly rotating Kerr black hole surrounded by an axially symmetric magnetic field homogeneous at infinity. They concluded that, if the magnetic field is present, the ISCO is located closer to the BH horizon. For the slowly rotating case they neglected terms involving $a^{2}$ in the metric (2.3), obtaining the following line element

$$
d s^{2}=-\left(1-\frac{2 M}{r}\right) d t^{2}-\frac{4 a M \sin ^{2} \theta}{r} d t d \theta+\left(1-\frac{2 M}{r}\right)^{-1} d r^{2}+r^{2} d \theta^{2}+r^{2} \sin ^{2} \theta d \phi^{2} .
$$

For a particle immersed in an uniform magnetic field in a curved space, its Lagrangian is

$$
\mathscr{L}=\frac{1}{2} g_{\mu v} \dot{x}^{\mu} \dot{x}^{\nu}+\frac{q A_{\mu} \dot{x}^{\mu}}{m_{0}}
$$


where $A_{\mu}$ is the electromagnetic 4-potential (which respects the Lorentz gauge $A_{; \mu}^{\mu}=0$ ). A special choice is $A_{\mu}=\left(\frac{2 a m B}{q}, 0,0, \frac{m B}{q}\right)$ [2]. Using this information, the solution for the geodesic equation in the equatorial plane $(\theta=\pi / 2)$ is

$$
\dot{t}=\frac{r^{3} E+2 a L}{r^{2}(r-2)}-2 a B, \quad \dot{\phi}=\frac{1}{r^{2}}\left(\frac{2 a E}{r-2}+L\right)-B .
$$

The $\dot{r}$ geodesic is expressed in terms of the plot of the potential in Fig. 7. In this same figure it is showed the escape velocity (i.e. $\dot{r}$ for the limit of the unbound motion).
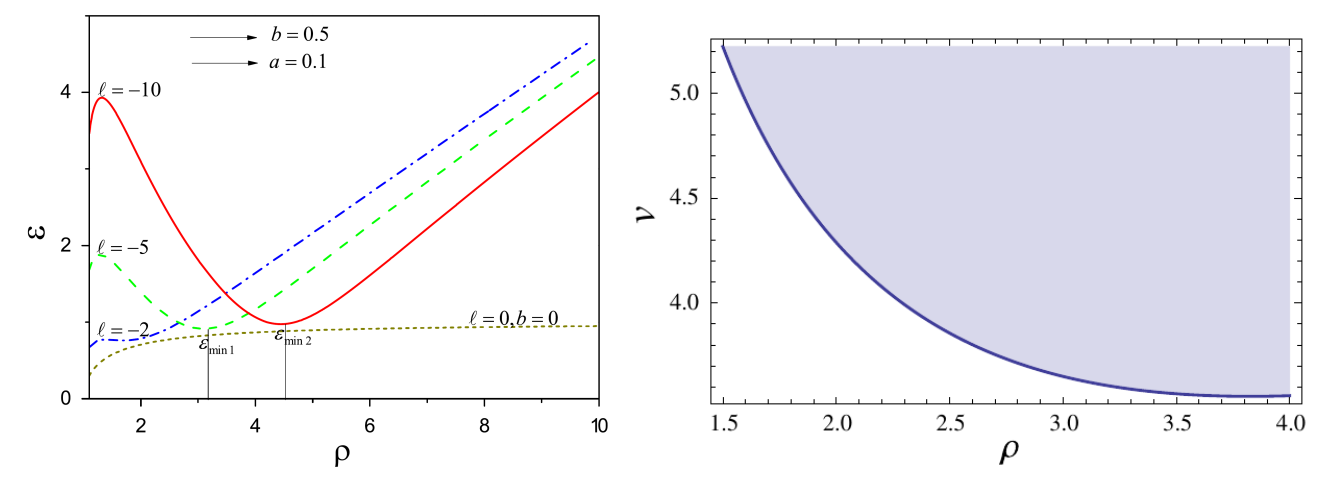

Figure 7: Slowly Kerr BHs with magnetic fields: the effective potential against radial coordinate $\rho$ for different values of angular momentum and the escape velocity of a particle in the vicinity of the horizon. In both cases, $a=0.1$. From [18].

\subsection{Charged particles orbiting magnetized rapid rotating Kerr black holes}

The presence of magnetic fields combined with higher black hole spins could imply in possible higher levels of particle acceleration. Nevertheless, a great amount of axial magnetic flux could also damp the motion of the charged particles.

The environment around a rapid rotating black hole can be described by the Kerr line element (2.3). Also, one can use the same Lagrangian (4.5) for describing the motion of charged particles immersed in an uniform magnetic field in curved space. In this case, from the geodesic equation solved at the equator, the center-of-mass energy is calculated as [13]

$$
E_{c . m .}=m_{0} \sqrt{2} \sqrt{1-g_{\mu v} u_{(1)}^{\mu} u_{(2)}^{v}},
$$

where

$$
\begin{gathered}
g_{\mu v} u_{(1)}^{\mu} u_{(2)}^{v}=\left(\frac{2}{r}-\frac{a^{2}}{r^{2}}-1\right) \frac{T_{1} T_{2}}{\Delta^{2}}+\frac{\left(a E_{1}-L_{1}\right)\left(a E_{2}-L_{2}\right)}{r^{2}}+\frac{r^{2}}{\Delta} \dot{r}_{(1)} \dot{r}_{(2)}, \\
E_{1}=E_{2}=1-2 a B+2 a B / r, \\
L_{1}=\ell_{1}+2 a^{2} B / r-\left(r^{2}+a^{2}\right) B, \quad L_{2}=\ell_{2}+2 a^{2} B / r-\left(r^{2}+a^{2}\right) B,
\end{gathered}
$$




$$
T_{i}=E_{i}\left(r^{2}+a^{2}\right)-L_{i} a .
$$

The radial geodesics $\dot{r}_{(i)}$ are obtained by solving the condition $g_{\mu \nu} u^{\mu} u^{\nu}=-1$. Figs. 8, 9 and 10 shows that the greater the spin $a$, the closer to the horizon the ISCO is. In the critical case, a weakly magnetized BH grants the bound state for the particle. As showed by [19] and [26], the $\mathrm{BH}$ spin and the intensity of the magnetic fields are correlated and they classify the Kerr BH as an accelerator candidate by the analysis of three possible domains: small $B$ 's and great $a$ 's, small $a$ 's and great $B$ 's and great values of $B$ 's and $a$ 's.

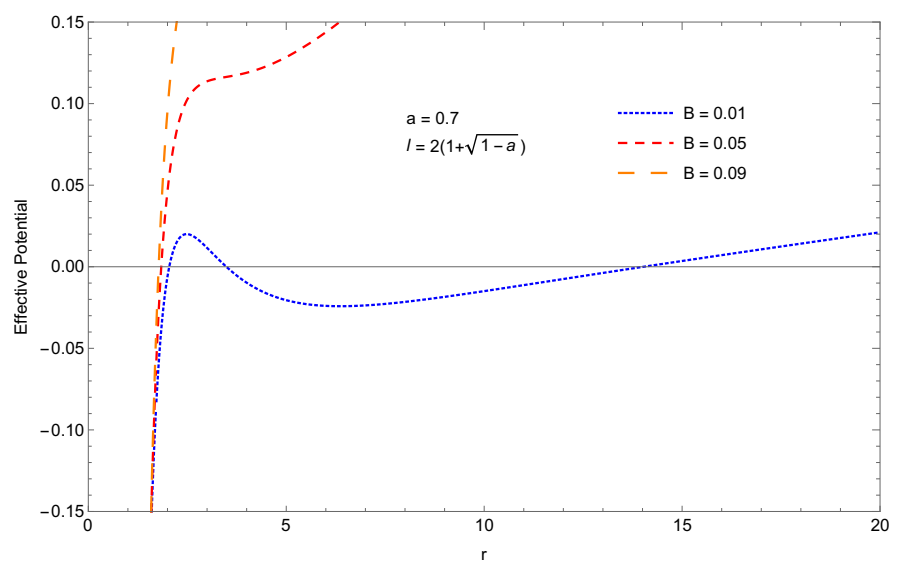

Figure 8: Rapid Kerr BHs with magnetic fields: the effective potential against radial coordinate for different values of magnetic fields $(a=0.99)$. From [13]. In the present case, the axial magnetic field is weaking the stability of the ISCO.

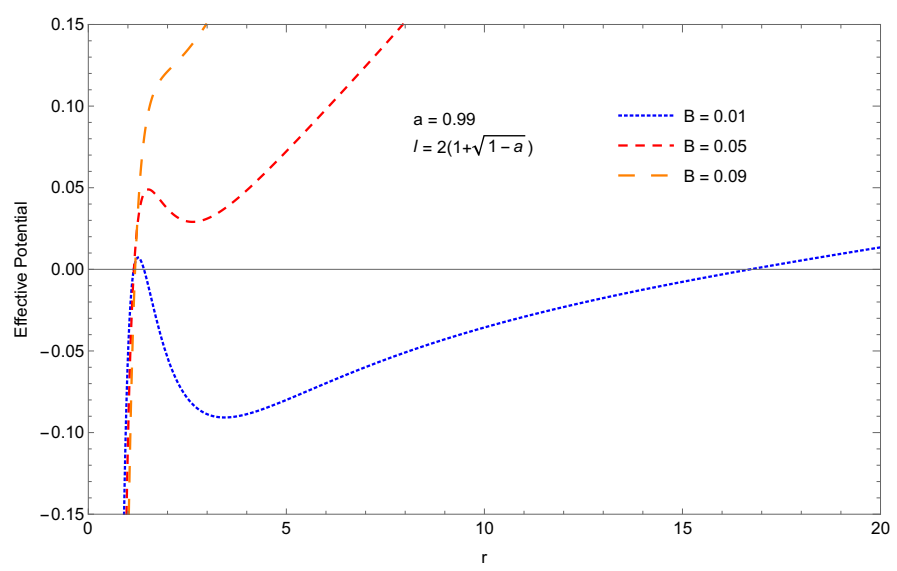

Figure 9: Rapid Kerr BHs with magnetic fields: the effective potential against radial coordinate for different values of magnetic fields $(a=0.7)$. From [13]. In the present case, the axial magnetic field is weaking the stability of the ISCO.

\section{Final remarks}

Literature points that ultra high energy cosmic rays (UHECRs) are produced by one mecha- 


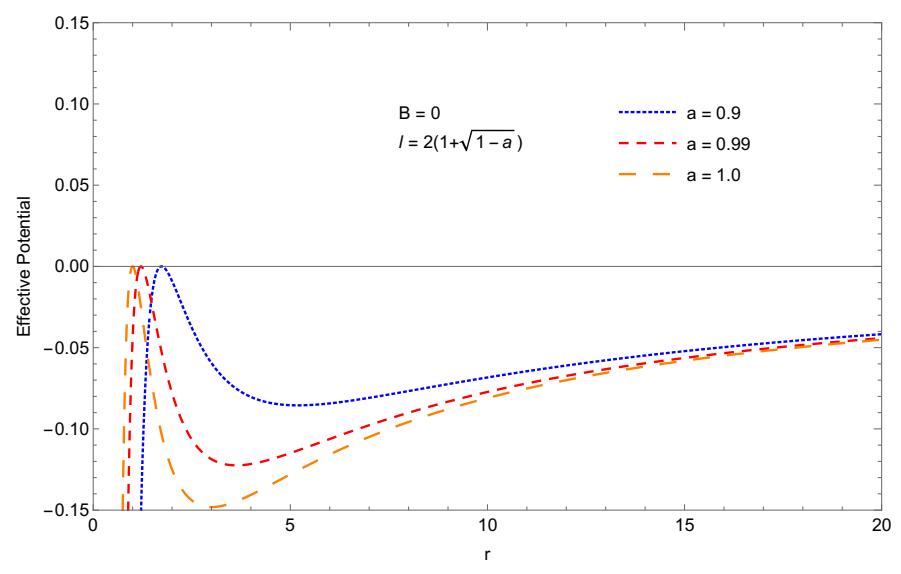

Figure 10: Rapid Kerr BHs with magnetic fields: the effective potential against radial coordinate for different values of magnetic fields (critical BH spins). From [13].

nism or a combination of high energy mechanisms (as shocks, unipolar induction, magnetic reconnection, etc). However, the previous sections show that BHs can work as accelerators of particles up to high energy. Nevertheless this is possible only if there are quite a few conditions, or even fine-tuning in the particle angular momentum, in the magnetic field and in the BH spin. Or explicitly, rapidly rotating BHs $(a \rightarrow 1)$ can accelerate particles to arbitrarily high energy if the angular momentum of the particle is fine-tuned to some critical value. But could it be possible that astrophysical Kerr BHs are distighished by such a large spin? Literature points to some supermassive BHs within AGNs with $a>0.99$ (see, e.g., [6]); nevertheless Thorne 1974 [27] calculated that it is mandatory that $a<0.998$ for astrophysical BHs due to accretion upper limits. In any case, if Killing time is regarded as big as the age of the universe, then critical test particles could be as energetic as UHECRs. In complement, as pointed by Berti et al. 2009 [8], "frame dragging effects in Kerr BHs can in principle accelerate particle to high energies, but (1) astrophysical restrictions on the spin severely limit the maximum CM energies in the collisions; (2) just as radiative losses constrain the performance of particle accelerators, gravitational radiation and back-reaction constrain the maximum CM energy for collisions around Kerr".

Here it was showed that it appears that certain values of fine-tuned axial magnetic fields also could help in the process of acceleration (only in case of critical $a$ 's). Otherwise, axial magnetic fields could cause a damping effect.

Other interesting investigations can be listed. For examples, the study of particles accelerated by charged rotating black holes (see [1, 15]). For further theoretical or phenomenological discussions about the energetic role of the central Kerr BH in the the propagation of UHECRs from AGNs, see [10, 11, 12, 3, 4]

\section{References}

[1] F. Ahmed, M. Amir, S. G. Gosh, arXiv 1805.00804.

[2] A. N. Aliev, N. O. Ozdemir, Mon. Not. R. Astron. Soc. 336, 241 (1978).

[3] R. C. Anjos, C. H. Coimbra-Araújo, R. da Rocha, V. Souza, JCAP 03, 014 (2016). 
[4] R. C. Anjos, C. H. Coimbra-Araújo, Phys. Rev. D 96, 023008 (2017).

[5] C. Bambi, Black Holes: A Laboratory for Testing Strong Gravity, Springer (2017).

[6] C. Bambi, Rev. Mod. Phys. 89, 025001 (2017).

[7] M. Bañados, J. Silk, S. M. West, Phys. Rev. Lett. 103, 111102 (2009).

[8] E. Berti et al., arXiv 0911.2243.

[9] R. D. Blandford, R. L. Znajek, Mon. Not. R. Astron. Soc. 179, 433 (1977).

[10] C. H. Coimbra-Araújo, R. C. Anjos, Phys. Rev. D 92, 103001 (2015).

[11] C. H. Coimbra-Araújo, R. C. Anjos, Clas. Quantum Grav. 33(18), 185010 (2016).

[12] C. H. Coimbra-Araújo, P. S. Letelier, Clas. Quantum Grav. 25(1), 015001 (2008).

[13] C. H. Coimbra-Araújo, R. C. Anjos, in preparation.

[14] A. M. El-Batal et al., Astroph. J. Lett. 826, L12 (2016).

[15] S. G. Gosh, R Sheoran, M. Amir, Phys. Rev. D 90, 103006 (2014).

[16] T. Harada, M. Kimura. Clas. Quantum Grav. 31243001 (2014).

[17] A. M. Hillas, The origin of ultra-high-energy cosmic rays. Ann. Rev. Astron. Astrophys. 22, 425 (1984).

[18] S. Hussain, I. Hussain, M. Jamil, Dynamics of a charged particle around a slowly rotating Kerr black hole immersed in magnetic field. Eur. Phys. J. C 74, 3210 (2014).

[19] T. Igata, T. Harada, M. Kimura, Phys. Rev. D 85, 104028 (2012).

[20] J. Linsley, Evidence for a primary cosmic-ray particle with energy $10^{20} \mathrm{eV}$, Phys. Rev. Lett. 10, 146-148 (1963).

[21] J. C. Mckinney, R. Narayan, Mon. Not. R. Astron. Soc. 375, 523 (2007).

[22] C. Misner, K. Thorne, J. Wheeler, Gravitation, Freeman \& Co (1973).

[23] K. Murase, C. D. Dermer, H. Takami, G. Migliori, Blazars as ultra-high-energy cosmic-ray sources: implications for TeV gamma-ray observations. Astrophys. J. 749, 63 âĂŞ78 (2012).

[24] M. L. Parker et al., Astroph. J. Lett. 821, L6 (2016).

[25] S. L. Shapiro, S. A Teukolsky, Black Holes, White Dwarfs and Neutron Stars: The Physics of Compact Objects, Wiley (1983).

[26] R. Shiose, M. Kimura, T. Chiba, Phys. Rev. D 90, 124016 (2014).

[27] K. S. Thorne, Astrophys. J, 191, 507 (1974).

[28] R. Znajek, Nature 262, 270 (1976). 